\title{
Macroporous Hydrogels for Stable Sequestration and Sustained Release of Vascular Endothelial Growth Factor and Basic Fibroblast Growth Factor Using Nucleic Acid Aptamers
}

\author{
Lidya Abune, Nan Zhao, Jinping Lai, Benjamin Peterson, Spencer Szczesny, Yong Wang* \\ Department of Biomedical Engineering, The Pennsylvania State University, University Park, PA \\ 16802, USA
}

\begin{abstract}
Macroporous hydrogels have been widely studied for biological and biomedical applications such as drug delivery and tissue engineering. However, these hydrogels cannot stably sequester molecules of interest due to their high permeability. The purpose of this work was to study the feasibility of using two aptamers to sequester two protein drugs, quantify the apparent diffusivity of protein drugs in aptamer-functionalized macroporous hydrogels, and evaluate the function of aptamer-functionalized macroporous hydrogels in controlling protein release for angiogenesis. Vascular endothelial growth factor (VEGF) and basic fibroblast growth factor (bFGF) were used as model proteins. The data show that anti-VEGF and anti-bFGF aptamers could be uniformly incorporated into macroporous hydrogels for stable and specific sequestration of VEGF and bFGF. The aptamers could reduce the apparent diffusivity of VEGF and bFGF in the macroporous hydrogels by approximately three orders of magnitude. Moreover, as the aptamers could prolong the release of these growth factors, dual aptamer-functionalized macroporous hydrogels could stimulate synergistic angiogenesis. Therefore, this work has successfully demonstrated that aptamer-functionalized macroporous hydrogels hold great potential of stably sequestering multiple molecules of interest for various biological and biomedical applications.
\end{abstract}

\section{Graphical Abstract}

\footnotetext{
*Correspondence can be addressed to Dr. Yong Wang (yxw30@psu.edu; Phone: 814-865-6867).

Supporting Information Available

The following files are available free of charge:
} 

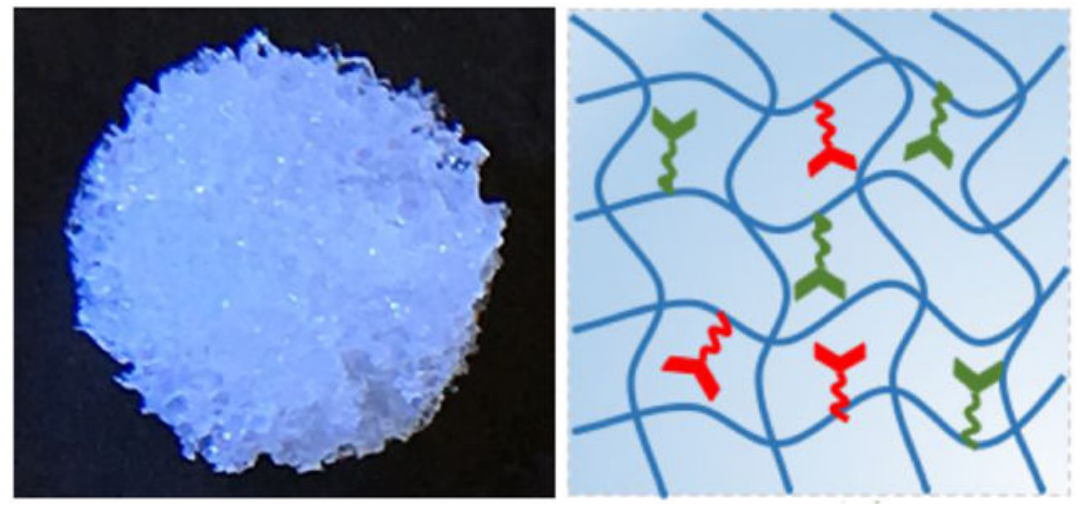

\section{Keywords}

macroporous hydrogel; protein delivery; growth factor; angiogenesis; aptamer

\section{Introduction}

Macroporous hydrogels have been studied for various applications such as drug delivery, tissue engineering, cancer therapy, biological separations, etc. ${ }^{1-6}$ Macroporous structures are needed for those applications as they allow for free molecular transport. ${ }^{7-9}$ For instance, when macroporous hydrogels are used for drug delivery, free transport of drugs allows the easy drug absorption after the synthesis of hydrogels. ${ }^{10}$ Thus, harsh reaction conditions will not cause the loss of the bioactivity of drugs. When macroporous hydrogels are used for tissue engineering, the macroporous structures allow for free transport of nutrients, oxygen, and wastes and easy infiltration and migration of cells within the macroporous structures. ${ }^{3}$ However, as the macroporous structures lead to high permeability, these hydrogels cannot hold molecules of interest (e.g., therapeutic proteins) within the cross-linked networks. Loaded molecules can be released abruptly. Therefore, macroporous hydrogels need to be functionalized to ensure stable sequestration and sustained release of specific molecules in numerous biological and biomedical applications.

Great efforts have been made to develop different strategies to functionalize hydrogels for affinity-based sequestration and release of biomolecules (e.g., proteins). ${ }^{11,12}$ For instance, heparin has been used to functionalize hydrogels as it is a linear polysaccharide with prevalent sulfate and carboxylate groups that allow heparin to bind to cationic proteins based on electrostatic interactions. ${ }^{13,14}$ Similarly, peptides have been applied to functionalize hydrogels for controlled protein release. ${ }^{15}$ Different from these elegant strategies, our group has applied nucleic acid aptamers to functionalize hydrogels for controlled protein release with the following reasons. ${ }^{16-18}$ Aptamers have high binding affinities and specificities with the ability to differentiate targets by subtle structural differences. ${ }^{19,20}$ Moreover, aptamers have tunable stability in biological environments and are tolerant of harsh thermal, physical, and chemical conditions. ${ }^{21}$ Aptamers also have little immunogenicity or toxicity as their compositions are nucleotides. ${ }^{22}$ Thus, aptamers have been used in different applications including not only hydrogel functionalization, ${ }^{16}$ but also cancer therapy, ${ }^{23,24}$ trigger design, ${ }^{25}$ and biosensor development. ${ }^{26}$ 
Our previous studies have demonstrated that aptamers are promising for the functionalization of hydrogels. ${ }^{16,27}$ In particular, we have demonstrated that aptamers are capable of effectively sequestering growth factors within macroporous hydrogels, ${ }^{18}$ and that aptamers can control the on-demand release of growth factors from macroporous hydrogels while maintaining their bioactivity. ${ }^{28,29}$ However, several critical issues were still not addressed, including the feasibility of functionalizing macroporous hydrogels with two aptamers, the quantitative analysis of apparent diffusivity of proteins in macroporous hydrogels, and the effect of dual protein release on biological systems. The purpose of this work was to address these questions. Specifically, we synthesized macroporous polyethylene glycol (PEG) hydrogels and functionalized them with two different aptamers. The two aptamers can bind to vascular endothelial growth factor (VEGF) and basic fibroblast growth factor (bFGF), respectively. With these two aptamers, we examined the capability of macroporous hydrogels in sequestering and releasing dual growth factors and calculated their apparent diffusivity using two different diffusion models. Additionally, the capability of dual aptamer-functionalized hydrogels in stimulating human umbilical vein endothelial cells (HUVECs) migration and chicken chorioallantoic membrane (CAM) vascularization was evaluated.

\section{Materials and Methods}

\subsection{Materials}

Poly (ethylene glycol) diacrylate (PEGDA; average Mn: 700 Da), and Pluronic F-127 were purchased from Sigma-Aldrich (St.Louis, MO). Phosphate buffered saline (PBS), ammonium persulfate (APS), Tetramethylethylenediamine (TEMED), and Sodium bicarbonate were obtained from Fisher Scientific (Pittsburgh, PA). Customized DNA nucleotide sequences were purchased from Integrated DNA Technologies (Coralville, IA). HUVECs, Medium 200 (M200), low serum growth supplement (LSGS), fetal bovine serum (FBS), and calcein AM were purchased from Thermofisher (Waltham, MA). Recombinant human vascular endothelial growth factor-165 (VEGF, MW = 38.2 kDa) and Recombinant Human fibroblast growth factor-basic (bFGF, MW = 16.4 kDa) and their respective enzymelinked immunosorbent assay (ELISA) Kit were purchased from PeproTech (Rocky Hill, NJ).

\subsection{Synthesis of aptamer-functionalized hydrogels}

Macroporous hydrogels were synthesized via free radical polymerization reaction coupled with gas formation reaction. ${ }^{30}$ In brief, 2\% (w/v) Pluronic F-127, 2\% (v/v) TEMED, 3\% $(\mathrm{w} / \mathrm{v})$ APS, $20 \%(\mathrm{v} / \mathrm{v})$ PEGDA, $4 \%(\mathrm{v} / \mathrm{v})$ acetic acid and deionized water were mixed. The mixed gel solution $(7 \mu \mathrm{L})$ was added to a cylindrical mold containing $50 \mathrm{mg}$ of sodium bicarbonate. For aptamer-functionalized macroporous hydrogels, acrylate modified aptamers were added to the gel solution prior to placing it into the cylindrical mold. Next, hydrogels were placed in deionized water overnight to remove unreacted molecules, sterilized in $70 \%$ ethanol for 1 hour and stored in Dulbecco's phosphate-buffered saline (DPBS) at $4{ }^{\circ} \mathrm{C}$ until use. 


\subsection{Assessment of hydrogel porosity and aptamer functionalization}

For Scanning Electron Microscope (SEM) characterization, hydrogels were frozen in $-80^{\circ} \mathrm{C}$ overnight, lyophilized, and sputter coated with gold. Hydrogels were imaged under an environmental scanning electron microscope (FEI Quanta 200, Hillsboro, OR). The pore size of the hydrogel was calculated using ImageJ Software. The average of the longest and the shortest diameter of the orifice of the pore was measured and taken as the pore diameter. To determine the pore volume of the hydrogel, water was loaded to a dehydrated hydrogel until the hydrogel sample was saturated. The porosity was estimated by dividing the volume of absorbed water to the total volume of the cylindrical hydrogel. Dual aptamerfunctionalized hydrogels were incubated with FAM-labeled Anti-VEGF (A-VEGF) and Cyanine-5 (Cy-5) labeled Anti-bFGF (A-bFGF) complementary oligonucleotide sequence and washed thoroughly to remove unbound oligonucleotides. The fluorescence images of bulk hydrogel were taken with a Maestro In Vivo Imaging System (Woburn, MA) and the higher magnification fluorescence images were taken using a scanning laser confocal microscope (Olympus FV1000, Centre Valley, PA).

\subsection{Prediction of secondary structures}

A-VEGF and A-bFGF aptamers secondary structure was generated by using the RNAstructure program, version 6.0.1 (https://rna.urmc.rochester.edu/RNAstructure.html). The program can be used for both RNA and DNA secondary structure prediction. From the predicted structures, the ones with the lowest free energy level were selected.

\subsection{Loading of growth factor}

Lyophilized VEGF and bFGF were reconstituted in M200 medium with $0.5 \%$ BSA. Before loading, hydrogels were dehydrated by gently blotting with sterilized tissue paper. A $20 \mu \mathrm{L}$ of the growth factor solution was then added to each hydrogel. The loaded hydrogel was then stored at $4^{\circ} \mathrm{C}$ for $24 \mathrm{hrs}$.

\subsection{Examination of growth factor sequestration}

Growth factor was loaded to hydrogels as stated. The growth factor to aptamer molecular ratio was 1:5. After loading, the hydrogels were placed in $1 \mathrm{~mL}$ of release medium (M200 with $0.5 \% \mathrm{BSA}$ ) at $37^{\circ} \mathrm{C}$ with a shaking rate of $70 \mathrm{rpm}$ for $4 \mathrm{hrs}$. The released supernatant was collected and stored at $-20^{\circ} \mathrm{C}$ until quantification.

\subsection{Examination of growth factor release}

Growth factor loaded hydrogels were incubated in $1 \mathrm{~mL}$ release medium (M200 with 0.5\% $\mathrm{BSA}$ ) at $37^{\circ} \mathrm{C}$ and $70 \mathrm{rpm}$ on a shaker. At the specific time points, the supernatant was totally collected and replaced with $1 \mathrm{~mL}$ of fresh medium. The collected supernatant was stored at $-20^{\circ} \mathrm{C}$ until quantification.

\subsection{Quantification of growth factors}

VEGF and bFGF were quantified using a recombinant human VEGF and bFGF enzymelinked immunosorbent assay (ELISA) kit respectively following the instructions provided by the manufacturer (Peprotech, Rocky Hill, NJ). Before analysis, the samples were diluted 
with the recommended diluent to ensure that the growth factors concentration fell within the detectable range of the assay. The absorbance of each sample was measured using an Infinite M200 Pro microplate reader (Tecan; Grodig, Austria) at $405 \mathrm{~nm}$ and was referenced by subtracting the absorbance at the reference wavelength $(650 \mathrm{~nm})$.

\subsection{Calculation of apparent diffusivity}

Diffusivity of growth factors in aqueous solution at $37^{\circ} \mathrm{C}$ was calculated using the StokesEinstein equation,

$$
D_{o}=\frac{K_{B} T}{6 \pi r \eta}
$$

where $\mathrm{D}_{\mathrm{o}}$ is the diffusivity of a protein in an aqueous solution, $\mathrm{r}$ is its hydrodynamic radius, $\mathrm{K}_{\mathrm{B}}$ is the Boltzman constant, $\mathrm{T}$ is temperature and $\eta$ is viscosity at $\mathrm{T}$. The hydrodynamic radius of bFGF and VEGF was estimated as $2 \mathrm{~nm}$ and $2.8 \mathrm{~nm}$, respectively, based on the proteins' molecular weight. ${ }^{31}$

The growth factor release profiles were fitted to calculate diffusion coefficients based on Fick's second law of diffusion for a cylindrical model. The assumptions include an infinite mass transfer coefficient in the boundary layer, a uniform initial drug distribution in the hydrogels, and perfect sink conditions. MatLab was used for the implementation of the two models.

Calculation with a semi-empirical equation.-The apparent diffusion coefficients for bFGF and VEGF from macroporous cylindrical hydrogels were analyzed via the semiempirical diffusion equation for a short-time release: ${ }^{32}$

$$
\frac{M_{t}}{M_{o}}=k t^{n}
$$

Where $\mathrm{M}_{\mathrm{t}}$ is the amount of released protein at time $\mathrm{t}, \mathrm{M}_{\mathrm{o}}$ is the initial amount of protein, $\frac{M_{t}}{M_{o}}$ is the fractional release, $\mathrm{t}$ is time, $\mathrm{n}$ is the diffusional exponent $(\mathrm{n}=0.45)$, and $\mathrm{k}$ is a kinetic constant and $k=4\left(\frac{D}{\pi R^{2}}\right)^{0.45}$, where $\mathrm{D}$ is the apparent diffusivity and $R$ is the radius of the hydrogel.

Calculation from a 2D Model.-We considered both radial and axial mass transfer. The apparent diffusion coefficients for bFGF and VEGF were analyzed via the diffusion equation: ${ }^{33}$ 


$$
\begin{aligned}
& \frac{M_{t}}{M_{o}}=1-\frac{32}{\pi^{2}} \sum_{n=1}^{\infty} \frac{1}{q_{n}^{2}} \exp \left(-\frac{q_{n}^{2}}{R^{2}} D t\right) \sum_{m=0}^{\infty} \frac{1}{(2 m+1)^{2}} \exp ( \\
& \left.-\frac{(2 m+1)^{2} \pi^{2}}{H^{2}} D t\right)
\end{aligned}
$$

Where $\mathrm{M}_{\mathrm{t}}$ is the amount of released protein at time t, $\mathrm{M}_{\mathrm{o}}$ is the initial amount of protein, $\frac{M_{t}}{M_{o}}$ is the fractional release, $\mathrm{D}$ is the apparent diffusivity, $\mathrm{t}$ is time, and $\mathrm{R}$ and $\mathrm{H}$ are radius and height of the cylinder, respectively, and $\mathrm{q}_{\mathrm{n}}$ represents the roots of the Bessel function of the first kind of order zero: $\mathrm{J}_{0}\left(\mathrm{q}_{\mathrm{n}}\right)=0$.

\subsection{Evaluation of cell migration using HUVECs}

HUVECs were cultured on 24-well plate in basal medium (M200+2\% LSGS). After confluence, HUVECs were starved with a low serum medium for $24 \mathrm{hrs}$. (M200+1\%FBS). HUVECs monolayer was scratched horizontally and vertically with a pipette tip to obtain a monolayer culture with space without cells. Cellular debris was removed by gently washing twice with PBS and cells were cultured in low serum medium.

Growth factor loaded hydrogels (Blank (100 ng bFGF+ 100 ng VEGF loaded), A-VEGF (200 ng VEGF loaded), A-bFGF (200 ng bFGF loaded), and Dual (100 ng bFGF+ 100 ng VEGF)) were placed in a release medium for 3 consecutive days. The hydrogels were then gently washed with DPBS and placed in an upper chamber by using transwell to further release growth factors to stimulate HUVECs. The scraped line was imaged using a fluorescence phase-contrast inverted microscope (Olympus IX73, Pittsburgh, PA) before and after incubation with hydrogels at $37^{\circ} \mathrm{C}$ for $24 \mathrm{hrs}$. Cell migration distance was analyzed using Image J Software.

\subsection{Examination of angiogenesis in CAM}

The chorioallantoic membrane (CAM) assay was used to determine the dual stimulation of VEGF and bFGF released from aptamer-functionalized hydrogels. Day 3 embryo fertile chicken eggs (E3) were obtained from The Poultry Education and Research Center at Penn State (PERC). The eggs were cracked, and embryos were placed on square weigh boats under sterile conditions. Then the embryos were incubated for 5 days at $38{ }^{\circ} \mathrm{C}$ and $4 \% \mathrm{CO}_{2}$ in a humidified incubator. On the 8th day (E8), samples were implanted on the CAM. The samples consisted of four groups: Blank hydrogel, A-VEGF functionalized hydrogel, AbFGF functionalized hydrogel and Dual aptamer-functionalized hydrogel. Prior to implantation, all hydrogels were loaded with growth factors similar to the in vitro study and placed in a release medium for 3 days. After implantation, the embryos were incubated until day 11 (E11). On day 11, the hydrogel implants were photographed with CRI Master In Vivo Imaging System. Within each treatment group, the number of new radial blood vessels formed around the implant during treatment period was counted to assess blood vessel formation. ${ }^{34}$ Four samples were analyzed per group $(\mathrm{N}=4)$. 
For microscopic analysis, the CAM was fixed in formalin, dehydrated through ethanol and embedded in paraffin wax. Paraffin-blocked tissues were sectioned into $6 \mu \mathrm{m}$ thick slices and stained with a Leica Autostainer (Buffalo Grove, IL). The Hematoxylin and Eosin (H \& E) stained samples were examined and photographed with Olympus IX5-S8F2 microscope (Centre Valley, PA).

\subsection{Statistical Analysis}

SPSS Statistics 25 (IBM Corp) was used for statistical analysis. For all experiments, three samples were analyzed per group $(\mathrm{N}=3)$ unless specified otherwise. One-way analysis of variance (ANOVA) followed by the Bonferroni post-test was performed for the comparison of multiple groups. The result was considered statistically significant if $\mathrm{p}<0.05$. Quantitative data are reported as mean \pm the standard deviation.

\section{Results and Discussion}

\subsection{Synthesis and structural characterization of dual aptamer-functionalized macroporous hydrogels}

Macroporous hydrogels can be developed with numerous methods including lyophilization, cryogelation, 3D printing, phase separation, and gas production. ${ }^{35-38}$ Each method has its advantages and disadvantages. In this work, we used gas production to develop macroporous structures for three major reasons. First, the formation of pores is instantaneous. Second, it does not need special instruments. Third, it is easy to control as the formation of pores is not determined by heat transfer.

Specifically, the aptamer-functionalized macroporous PEG hydrogel was synthesized using free radical polymerization coupled with the production of carbon dioxide. Aptamers were conjugated with an acrylate group at their 5' ends followed by a poly-A sequence to serve as a spacer to eliminate potential steric hindrance for the binding of aptamers to growth factors. As the aptamers had the acrylate group, A-bFGF and A-VEGF aptamers were conjugated to the PEG network during free radical polymerization (Fig. 1.A). This polymerization technique allows aptamers to be chemically conjugated to the hydrogel and serve as binding sites for growth factors. For the formation of macroporous pores, acetic acid was added to the pre-gelation solution and allowed to react with sodium bicarbonate placed in the cylindrical mold. Consequently, polymerization coupled with $\mathrm{CO}_{2}$ formation would allow for the formation of large pores in the PEG hydrogels.

Indeed, the images show that the hydrogels had macroporous structures (Fig. 1.B\&C). The porous hydrogel was able to absorb $\sim 30 \mu \mathrm{L}$ of water, which is equivalent to $75 \%$ porosity. Moreover, the SEM images show that the pore structure remained the same before and after single (A-VEGF or A-bFGF) or dual (A-VEGF and A-bFGF) aptamer incorporation, suggesting that the incorporation of multiple aptamers has no significant effect on the macroporous structure of hydrogels. Blank, A-VEGF, A-bFGF and dual aptamer functionalized hydrogels have an average pore size of $52 \pm 18 \mu \mathrm{m}, 51 \pm 19 \mu \mathrm{m}, 56 \pm 17 \mu \mathrm{m}$, and $51 \pm 19 \mu \mathrm{m}$, respectively (Fig S1). 
To examine aptamer incorporation, fluorescence and confocal images of aptamerfunctionalized hydrogels were captured after the hydrogels were stained with FAM-labeled A-VEGF and Cy-5 labeled A-bFGF complementary sequences. As shown in Fig. 1D, the strong green and red fluorescent signals from the whole hydrogel suggest the successful incorporation of anti-VEGF and anti-bFGF aptamers to the macroporous hydrogel. Confocal microscopy was utilized to further analyze the distribution of two aptamers in the hydrogel network. The confocal microscopy image confirmed the successful incorporation and the relatively uniform distribution of aptamers in the macroporous hydrogel network (Fig. 1D).

\subsection{Examination of growth factor sequestration}

After confirming that dual aptamers could be successfully conjugated to macroporous hydrogels, we studied the sequestration of growth factors in aptamer-functionalized macroporous hydrogels. The secondary structures of A-VEGF and A-bFGF are shown in Fig. 2A. A-VEGF has 26 nucleotides with a high affinity to VEGF and can sequester VEGF effectively. ${ }^{39}$ A-bFGF has 32 nucleotides with a high affinity to $\mathrm{BFGF}^{40}$ Both aptamers were used to functionalize hydrogels in a molar ratio of five aptamers to one growth factor for the assessment of growth factor sequestration. Before loaded with growth factors (Fig. 2B), hydrogels were thoroughly washed to remove any unreacted molecules including free aptamers. After washed, hydrogels were blotted for dehydration for the loading of growth factors. This process ensures growth factors to retain their activity or eliminate the possibility of denaturation or neutralization due to the reactants or by-products.

It is important to note that aptamer-functionalized macroporous hydrogels do not need special conditions such as low temperature for their storage or shipping as both aptamers and hydrogels are stable. Moreover, the synthesis of hydrogels and the loading of proteins are separated. Therefore, target proteins such as growth factors can be loaded into the macroporous hydrogel only when needed. This flexibility makes the transition of aptamerfunctionalized macroporous hydrogels from the laboratory to the clinic possible, allowing for the design of cost-effective, easy-to-use and off-the-shelf protein delivery biomaterials.

Fig. 2C shows that A-VEGF retained more than $90 \%$ of VEGF in the macroporous hydrogels. In contrast, Blank and A-bFGF-functionalized macroporous hydrogels retained $15 \%$ and $33 \%$ of VEGF, respectively. Moreover, dual aptamer-functionalized hydrogels retained the same amount of VEGF as the A-VEGF functionalized hydrogels. Similarly, AbFGF retained more than $95 \%$ of bFGF whereas Blank and A-VEGF hydrogels retained $23 \%$ and $34 \%$, respectively (Fig. 2D). Dual aptamer-functionalized hydrogels retained the same amount of bFGF as the anti-bFGF functionalized hydrogels. These data show that while macroporous hydrogels are highly permeable, aptamers can stably retain growth factors. Additionally, previous studies show that the amount of growth factor retained in the hydrogel increases as the aptamer concentration increases until it reaches a certain threshold.

${ }^{29}$ Retention is mainly determined by the binding affinity and density of aptamers. Moreover, aptamer-mediated growth factor retention is not significantly affected by the copresence of another aptamer in the same hydrogel system. However, it is good to note that there was slightly higher retention of non-target growth factors in aptamer-functionalized hydrogels compared to blank hydrogels. This retention might be attributed to the 
electrostatic interactions between aptamers and proteins. The ratio of growth factors to aptamers can be optimized to tune the retention of growth factors. Thus, while we used AVEGF and A-bFGF as examples to illustrate the capability of dual aptamer-functionalized hydrogels in sequestering two growth factors, it is possible that more than two aptamers can be incorporated into macroporous hydrogels for multiple aptamer-specific growth factor binding and retention.

\subsection{Examination of growth factor release from aptamer-functionalized macroporous hydrogels.}

In general, diffusion is the major mechanism of determining the release of growth factors from hydrogels that do not have specific interactions with loaded growth factors (Fig. 3A). However, when growth factor-specific aptamers are incorporated into hydrogels, release will be determined by not only diffusion but also aptamer-growth factor binding (Fig. 3A). In this work, we used apparent diffusivity to consider the overall effects of diffusion and binding reactions rather than separating them as individual factors in controlling the release procedure. Thus, the release data were directly fitted to two diffusion equations that were both originally established based on Fick's second law of diffusion. The diffusivity of bFGF and VEGF from blank, single aptamer and dual aptamer-functionalized cylindrical macroporous hydrogels were analyzed and compared.

The semi-empirical equation (Equation 2) has been widely used to fit the release profiles of drugs from polymeric systems with different geometries. In this equation, the effects of geometries on mass transfer is not specifically considered. For a thin planar material, this equation is largely effective to simulate $\sim 60 \%$ release of molecules. It is also largely valid for simulating $\sim 15 \%$ release from a material with a cylindrical or spherical geometry. To ensure that this semi-empirical equation has reasonable fidelity to simulate the major portion (e.g., $60 \%$ release) of the release process of cylindrical objects, the diffusional exponent $n$ was suggested to be corrected from 0.5 to $0.45 .{ }^{32}$ The diffusional exponent was set at 0.45 in this semi-empirical equation for our calculation and less than $60 \%$ release was fit to calculate the apparent diffusivity (Fig. 3B).

The calculation showed VEGF has apparent diffusivity of $(11 \pm 1.3) \times 10^{-10} \mathrm{~cm}^{2} / \mathrm{sec},(18$ $\pm 2.2) \times 10^{-10} \mathrm{~cm}^{2} / \mathrm{sec}$, and $(2.2 \pm 0.3) \times 10^{-7} \mathrm{~cm}^{2} / \mathrm{sec}$ in dual aptamer-functionalized, single aptamer-functionalized and blank hydrogels, respectively (Fig. 4A). Apparent diffusivity of bFGF was $(7.1 \pm 2.9) \times 10^{-10} \mathrm{~cm}^{2} / \mathrm{sec},(13 \pm 4.2) \times 10^{-10} \mathrm{~cm}^{2} / \mathrm{sec}$, and $(3.1$ $\pm 0.4) \times 10^{-7} \mathrm{~cm}^{2} / \mathrm{sec}$ in dual aptamer-functionalized, single aptamer-functionalized and blank hydrogels, respectively (Fig. 4A).

As the geometry of the cylindrical hydrogel is confined and all surfaces are important for mass transfer, the release of growth factors from the cylindrical hydrogel is actually a twodimensional molecular transport phenomenon. Particularly, the aspect ratio $(2 \mathrm{R} / \mathrm{H})$ was 2.5 for the cylindrical hydrogels as we synthesized. The side and base surface areas were significant in our system. Thus, in addition to using the semi-empirical equation to calculate the apparent diffusivity, we used Equation 3 to calculate the apparent diffusivity. The simulation well fits the experimental measurement (Fig. 3C). Through the curve fitting, the calculated apparent diffusivity of VEGF in dual and single aptamer-functionalized and blank 
hydrogels was $(4.8 \pm 0.2) \times 10^{-10} \mathrm{~cm}^{2} / \mathrm{sec},(7.7 \pm 0.3) \times 10^{-10} \mathrm{~cm}^{2} / \mathrm{sec}$, and $(2.5 \pm 0.7) \times$ $10^{-7} \mathrm{~cm}^{2} / \mathrm{sec}$, respectively (Fig. 4A). The apparent diffusivity of bFGF in dual and single aptamer-functionalized and blank hydrogel was $(1.2 \pm 0.1) \times 10^{-10} \mathrm{~cm}^{2} / \mathrm{sec},(2.7 \pm 0.1) \times$ $10^{-10} \mathrm{~cm}^{2} / \mathrm{sec}$, and $(1.0 \pm 0.1) \times 10^{-7} \mathrm{~cm}^{2} / \mathrm{sec}$, respectively (Fig. 4A). The apparent diffusivity values between single versus dual aptamer-functionalized hydrogel did not exhibit a huge difference. In comparison, the diffusivity calculated using the 2D model for both growth factors was lower than that from the semi-empirical equation.

Regardless of which equation we used, the experimental results show that with aptamers, the release of growth factors was slowed. Importantly, the apparent diffusivity of VEGF and bFGF was reduced by approximately three orders of magnitude in the presence of the aptamers (Figure 4B). These results show that aptamer functionalization is a powerful tool in altering the diffusivity of growth factors sequestered in macroporous hydrogels. Many factors such as the pore size can affect the diffusivity of growth factors in hydrogels. In general, the increase of the pore size will lead to the increase of apparent diffusivity. However, once hydrogels are functionalized with aptamers, the pore size is not a major factor in determining the apparent diffusivity of growth factors. Instead it is primarily determined by the density and binding affinity of aptamers. Thus, it is possible to tune apparent diffusivity of growth factors by modulating both binding affinity and density of aptamers to achieve specific release profiles to meet specific requirements in practical applications.

\subsection{Evaluation of dual aptamer-functionalized macroporous hydrogel in releasing growth factors for stimulating angiogenesis.}

To demonstrate potential applications of aptamer-functionalized macroporous hydrogels, we examined the effects of VEGF and/or bFGF release on the migration of endothelial cells and the growth of blood vessels (i.e., angiogenesis). Angiogenesis is an essential biological process in which new blood vessels develop from preexisting vasculature. It is a highly complex biological process that is not regulated by only a single growth factor but by numerous biomolecules. ${ }^{41}$ Stimulation of angiogenesis can be applicable for various biomedical applications including ischemic heart disease, wound healing, and peripheral and coronary vascular diseases. ${ }^{42-45}$

VEGF and bFGF are two of the most extensively studied prominent stimulants of angiogenesis. ${ }^{46}$ While the delivery of each growth factor can induce angiogenesis, it has been shown to yield transient angiogenesis. ${ }^{45,47}$ Presumably because of this reason, clinical trials using VEGF for the treatment of ischemic disease showed very limited efficacy in the stimulation of vasculature and the improvement of blood supply. ${ }^{45,47,48}$ It suggests that multiple growth factors are needed to stimulate the formation of a stable vasculature due to the complexity of the biological process.

The synergistic effects of VEGF and bFGF on angiogenesis has been studied. ${ }^{49-53}$ For example, the exposure of microvascular endothelial cells grown on 3D-collagen gels to VEGF and bFGF stimulated a better and faster cell response than individual growth factors. 53 The capillary-like sprouts stimulated by the combination of VEGF and bFGF was longer than the sum of their individual effects. ${ }^{50}$ These previous findings indicate that VEGF and 
bFGF function synergistically to enhance angiogenesis. The potential mechanisms responsible for this synergism are at least two-folded. First, bFGF can induce the expression of endogenous VEGF and its receptors in endothelial cells. ${ }^{54}$ Second, VEGF-induced expression of plasminogen activator that is a hallmark of VEGF activity depends on bFGF. ${ }^{55}$ Based on these critical findings, we were inspired to use VEGF and bFGF as a model to examine whether dual release of growth factors from aptamer-functionalized macroporous hydrogels would have therapeutic potential.

The capability of bFGF and VEGF for stimulating angiogenic response was assessed using both in vitro and in vivo assays. The in vitro assay was the cell migration of the endothelial monolayer. With this assay, we studied the migration of endothelial cells after an artificial wound was created.

The distance of cell migration before and after treatment with VEGF and/or bFGF was imaged and calculated. Fig. 5A shows that the cell migration distance was higher in the group of bFGF and VEGF release (Dual) than all the other three groups including the group of individual growth factor release. Fig. 5B quantitatively shows that the cell migration distance in the Dual group was $\sim 75 \%$ while it was $\sim 45 \%$ and $\sim 25 \%$ in the groups of individual bFGF and VEGF release, respectively. The cell migration distance in the blank hydrogel group was less than $10 \%$. Clearly, the results from the in vitro assay demonstrate that dual release of VEGF and bFGF can stimulate angiogenic response more effectively.

We further used the CAM model to demonstrate the effect of dual VEGF and bFGF release on angiogenesis in an in vivo setting. Fig. 5C shows the representative images captured from all treatment groups. Fig. 5D shows the quantitative analysis of the new blood vessel formation. The number of new blood vessel formed in the group of dual aptamerfunctionalized macroporous hydrogels loaded with both VEGF and bFGF was 3 times higher than that in the group of blank hydrogels loaded with VEGF and bFGF. The results also show that dual delivery led to the growth of more blood vessels than individual delivery (Fig.5D). We further stained the cross-section of CAM tissue treated with dual aptamerfunctionalized macroporous hydrogels loaded with VEGF and bFGF. The images indicate that the newly formed blood vessels surrounding the hydrogels had blood cells (Fig.5E), and that the newly formed vessels were biologically functional. Taken together, these results suggest that dual aptamer functionalized macroporous hydrogels have the capability of sequestering and releasing VEGF and bFGF for enhanced angiogenesis.

\section{Conclusions}

In summary, macroporous hydrogels can be functionalized with two different aptamers during polymerization. The aptamers can specifically and stably sequester corresponding growth factors. With aptamer-mediated stable sequestration, the apparent diffusivity of loaded growth factors in macroporous hydrogels can be reduced by several orders of magnitude. Resultantly, the release of growth factors from aptamer-functionalized macroporous hydrogels can be slowed and sustained. Moreover, dual aptamer-functionalized macroporous hydrogels can release two different growth factors for synergistic angiogenesis. While we have demonstrated macroporous hydrogels can be functionalized with two 
aptamers for sequestering and releasing two different growth factors, it is reasonable to anticipate that multiple aptamers and growth factors can be incorporated into the same macroporous hydrogels for growth factor delivery. In addition, complementary DNA triggers can be used to control on-demand release of multiple growth factors. This potential will be explored in our future work. It is further important to note that while we studied the promise of aptamer-functionalized macroporous hydrogels in promoting angiogenesis, aptamerfunctionalized macroporous hydrogels may find other applications such as cancer immunotherapy and biological separations.

\section{Supplementary Material}

Refer to Web version on PubMed Central for supplementary material.

\section{Acknowledgments}

Authors thank Ryan Chaky and The Huck Institute Microscopy Facilities (University Park, PA) for technical support and The Poultry Education and Research Center at Penn State for providing chicken embryos. Research reported in this work was supported in part by the National Institutes of Health (HL122311; AR073364). L.A. thanks the National Center for Advancing Translational Sciences, NIH Grants TL1 TR002016 and UL1 TR002014. (The content is solely the responsibility of the authors and does not necessarily represent the official views of the NIH.)

\section{References}

(1). Im P \& Kim J On-Demand Macroscale Delivery System Based on a Macroporous Cryogel with a High Drug Loading Capacity for Enhanced Cancer Therapy. ACS Biomater. Sci. Eng 4, 34983505 (2018). DOI: 10.1021/acsbiomaterials.8b00911.

(2). Patil NS, Dordick JS \& Rethwisch DG Macroporous poly(sucrose acrylate) hydrogel for controlled release of macromolecules. Biomaterials 17, 2343-2350 (1996). DOI: 10.1016/ S0142-9612(96)00089-0. [PubMed: 8982474]

(3). Fan C \& Wang D-A Macroporous Hydrogel Scaffolds for Three-Dimensional Cell Culture and Tissue Engineering. Tissue Eng. Part B Rev 23, 451-461 (2017). DOI: 10.1089/ten.teb. 2016.0465. [PubMed: 28067115]

(4). Ford MC, Lavik EB, Hynes SR, Young M, Segal SS, Madri JA, Bertram JP, Li Q and Michaud M A macroporous hydrogel for the coculture of neural progenitor and endothelial cells to form functional vascular networks in vivo. Proc. Natl. Acad. Sci 103, 2512-2517 (2006). DOI: 10.1073/pnas.0506020102. [PubMed: 16473951]

(5). Lewin SA, Shih T-Y, Li WA, Mooney DJ, Warren Sands R, Bencherif SA, Bhatta D, Ali OA, Braschler TM, Dranoff G and Verbeke CS Injectable cryogel-based whole-cell cancer vaccines. Nat. Commun 6, 1-13 (2015). DOI: 10.1038/ncomms8556.

(6). Ertürk G \& Mattiasson B Cryogels-versatile tools in bioseparation. J. Chromatogr. A 1357, 24-35 (2014). DOI: 10.1016/j.chroma.2014.05.055. [PubMed: 24915836]

(7). Lee Andrew G., Arena Christopher P., Beebe David J., and S. P. P. Development of Macroporous Poly(ethylene glycol) Channels. 11, 3316-3324 (2011). DOI: 10.1021/bm100792y.

(8). Amsden B Solute diffusion within hydrogels. Mechanisms and models. Macromolecules 31, 8382-8395 (1998). DOI: 10.1021/ma980765f.

(9). De France KJ, Xu F \& Hoare T Structured Macroporous Hydrogels: Progress, Challenges, and Opportunities. Adv. Healthc. Mater 7, 1-17 (2018). DOI: 10.1002/adhm.201700927.

(10). Li J \& Mooney DJ Designing hydrogels for controlled drug delivery. Nat. Rev. Mater 1, (2016). DOI: $10.1038 /$ natrevmats.2016.71.

(11). Bessodes M, Silva AKA, Scherman D, Merten O-W \& Richard C Growth Factor Delivery Approaches in Hydrogels. Biomacromolecules 10, 9-18 (2008). DOI: 10.1021/bm801103c. 
(12). Belair DG, Le NN \& Murphy WL Design of growth factor sequestering biomaterials. Chem. Commun 50, 15651-15668 (2014). DOI: 10.1039/c4cc04317k.

(13). Rabenstein DL Heparin and heparan sulfate: Structure and function. Nat. Prod. Rep 19, 312-331 (2002). DOI: 10.1039/b100916h. [PubMed: 12137280]

(14). Liang Y \& Kiick KL Heparin-functionalized polymeric biomaterials in tissue engineering and drug delivery applications. Acta Biomater. 10, 1588-1600 (2014). DOI: 10.1016/j.actbio. 2013.07.031. [PubMed: 23911941]

(15). Leslie-Barbick JE, Saik JE, Gould DJ, Dickinson ME \& West JL The promotion of microvasculature formation in poly(ethylene glycol) diacrylate hydrogels by an immobilized VEGF-mimetic peptide. Biomaterials 32, 5782-5789 (2011). DOI: 10.1016/j.biomaterials. 2011.04.060. [PubMed: 21612821]

(16). Battig MR, Soontornworajit B \& Wang Y Programmable release of multiple protein drugs from aptamer-functionalized hydrogels via nucleic acid hybridization. J. Am. Chem. Soc 134, 1241012413 (2012). DOI: 10.1021/ja305238a. [PubMed: 22816442]

(17). Zhang X, Battig MR \& Wang Y Programmable hydrogels for the controlled release of therapeutic nucleic acid aptamers via reversible DNA hybridization. Chem. Commun 49, 9600-2 (2013). $10.1039 / \mathrm{c} 3 \mathrm{cc} 45594 \mathrm{~g}$.

(18). Battig MR, Huang Y, Chen N \& Wang Y Aptamer-functionalized superporous hydrogels for sequestration and release of growth factors regulated via molecular recognition. Biomaterials 35 , 8040-8048 (2014). DOI: 10.1016/j.biomaterials.2014.06.001. [PubMed: 24954732]

(19). Lee JF, Stovall GM \& Ellington AD Aptamer therapeutics advance. Current Opinion in Chemical Biology 10, 282-289 (2006). DOI: 10.1016/j.cbpa.2006.03.015. [PubMed: 16621675]

(20). Proske D, Blank M, Buhmann R \& Resch A Aptamers - Basic research, drug development, and clinical applications. Applied Microbiology and Biotechnology 69, 367-374 (2005). DOI: 10.1007/s00253-005-0193-5. [PubMed: 16283295]

(21). Burmeister PE, Lewis SD, Silva RF, Preiss JR, Horwitz LR, Pendergrast PS, McCauley TG, Kurz JC, Epstein DM, Wilson C and Keefe AD Direct in vitro selection of a $2^{\prime}$-O-methyl aptamer to VEGF. Chem. Biol 12, 25-33 (2005). DOI: 10.1016/j.chembiol.2004.10.017. [PubMed: 15664512]

(22). Nimjee SM, Rusconi CP \& Sullenger BA Aptamers: An Emerging Class of Therapeutics. Annu. Rev. Med 56, 555-583 (2005). DOI: 10.1146/annurev.med.56.062904.144915. [PubMed: 15660527]

(23). Li J, Zheng H, Bates PJ, Malik T, Li XF, Trent JO and Ng CK Aptamer imaging with Cu-64 labeled AS1411: Preliminary assessment in lung cancer. Nucl. Med. Biol 41, 179-185 (2014). DOI: 10.1016/j.nucmedbio.2013.10.008. [PubMed: 24373858]

(24). Bates PJ, Laber DA, Miller DM, Thomas SD \& Trent JO Discovery and development of the Grich oligonucleotide AS1411 as a novel treatment for cancer. Experimental and Molecular Pathology 86, 151-164 (2009). DOI: 10.1016/j.yexmp.2009.01.004. [PubMed: 19454272]

(25). Ke Y, Lindsay S, Chang Y, Liu Y \& Yan H Self-assembled water-soluble nucleic acid probe tiles for label-free RNA hybridization assays. Science (80-.). 319, 180-183 (2008). DOI: 10.1126/ science. 1150082 .

(26). Huang CC, Huang YF, Cao Z, Tan W \& Chang HT Aptamer-modified gold nanoparticles for colorimetric determination of platelet-derived growth factors and their receptors. Anal Chem 77, 5735-5741 (2005). DOI: 10.1021/ac050957q. [PubMed: 16131089]

(27). Fan T-H, Soontornworajit B, Karzar-Jeddi M, Zhang X \& Wang Y An aptamer-functionalized hydrogel for controlled protein release: A modeling study. Soft Matter 7, 9326 (2011). DOI: 10.1039/c1sm05802a.

(28). Zhang X, Battig MR, Chen N, Gaddes ER, Duncan KL and Wang Y Chimeric Aptamer-Gelatin Hydrogels as an Extracellular Matrix Mimic for Loading Cells and Growth Factors. Biomacromolecules 17, 778-787 (2016). DOI: 10.1021/acs.biomac.5b01511. [PubMed: 26791559]

(29). Zhao N, Battig MR, Xu M, Wang X, Xiong N and Wang Y Development of a Dual-Functional Hydrogel Using RGD and Anti-VEGF Aptamer. Macromol. Biosci 17, 1-8 (2017). DOI: 10.1002/mabi.201700201. 
(30). Gemeinhart Richard A., Park Haesun \& Park Kinam. Pore Structure of Superporous Hydrogels. Polym. Adv. Technol 11, 617-625 (2000).

(31). Venturoli D \& Rippe B Ficoll and dextran vs. globular proteins as probes for testing glomerular permselectivity: effects of molecular size, shape, charge, and deformability. Am. J. Physiol. Physiol 288, F605-F613 (2005). DOI: 10.1152/ajprenal.00171.2004.

(32). Ritger PL \& Peppas NA a Simple Equation for Description of Solute Release 1. J. Control. Release, 5, 23-36 (1987).

(33). Siepmann J, Lecomte F \& Bodmeier R Diffusion-controlled drug delivery systems: Calculation of the required composition to achieve desired release profiles. J. Control. Release 60, 379-389 (1999). DOI: 10.1016/S0168-3659(99)00093-0. [PubMed: 10425342]

(34). Oktavia S, Wijayanti N \& Retnoaji B Anti-angiogenic effect of Artocarpus heterophyllus seed methanolic extract in ex ovo chicken chorioallantoic membrane. Asian Pac. J. Trop. Biomed 7, 240-244 (2017). DOI: 10.1016/j.apjtb.2016.12.024.

(35). Savina IN, Ingavle GC, Cundy AB \& Mikhalovsky SV A simple method for the production of large volume 3D macroporous hydrogels for advanced biotechnological, medical and environmental applications. Sci. Rep 6, 1-9 (2016). DOI: 10.1038/srep21154. [PubMed: 28442746]

(36). Chen J, Park H \& Park K Synthesis of superporous hydrogels: Hydrogels with fast swelling and superabsorbent properties. J. Biomed. Mater. Res 44, 53-62 (1999). DOI: 10.1002/ (SICI)1097-4636(199901)44:1<53::AID-JBM6>3.0.CO;2-W. [PubMed: 10397904]

(37). Ras RHA, Kettunen M, Ikkala O, Lindström T, Kemell M, Ruokolainen J, Nykänen A, Pore V, Houbenov N, Ankerfors M, Ritala M, Silvennoinen RJ and Sainio J Photoswitchable Superabsorbency Based on Nanocellulose Aerogels. Adv. Funct. Mater 21, 510-517 (2010). DOI: $10.1002 /$ adfm.201001431.

(38). Park A, Wu B \& Griffith LG Integration of surface modification and 3D fabrication techniques to prepare patterned poly(L-lactide) substrates allowing regionally selective cell adhesion. J. Biomater. Sci. Polym. Ed 9, 89-110 (1998). DOI: 10.1163/156856298X00451. [PubMed: 9493839]

(39). Coyne J, Davis B, Kauffman D, Zhao N \& Wang Y Polymer Microneedle Mediated Local Aptamer Delivery for Blocking the Function of Vascular Endothelial Growth Factor. ACS Biomater. Sci. Eng 3, 3395-3403 (2017). DOI: 10.1021/acsbiomaterials.7b00718. [PubMed: 29707631]

(40). Cannone JJ, Barnes CL, Achari A \& Kundrot CE Crystallization of bFGF-DNA aptamer complexes using a Sparse Matrix designed for protein-nucleic acid complexes. J. Cryst. Growth 232, 409-417 (2001). DOI: 10.1038/80430.

(41). Ucuzian AA, Gassman AA, East AT \& Greisler P J Burn Care Res. 31, 1-28 (2011). DOI: 10.1097/BCR.0b013e3181c7ed82.Molecular.

(42). Iyer SR \& Annex BH Therapeutic Angiogenesis for Peripheral Artery Disease: Lessons Learned in Translational Science. JACC Basic to Transl. Sci 2, 503-512 (2017). DOI: 10.1016/j.jacbts. 2017.07.012.

(43). Greaves NS, Ashcroft KJ, Baguneid M \& Bayat A Current understanding of molecular and cellular mechanisms in fibroplasia and angiogenesis during acute wound healing. J. Dermatol. Sci 72, 206-217 (2013). DOI: 10.1016/j.jdermsci.2013.07.008. [PubMed: 23958517]

(44). Zachary I \& Morgan RD Therapeutic angiogenesis for cardiovascular disease: Biological context, challenges, prospects. Heart 97, 181-189 (2011). DOI: 10.1136/hrt.2009.180414. [PubMed: 20884790]

(45). Al Sabti H Therapeutic angiogenesis in cardiovascular disease. J. Cardiothorac. Surg 2, 1-9 (2007). DOI: 10.1186/1749-8090-2-49. [PubMed: 17201932]

(46). Post MJ, Laham R, Sellke FW \& Simons M Therapeutic angiogenesis in cardiology using protein formulations. Cardiovasc. Res 49, 522-531 (2001). DOI: 10.1016/S0008-6363(00)00216-9. [PubMed: 11166265]

(47). Molin D \& Post MJ Therapeutic angiogenesis in the heart: protect and serve. Curr. Opin. Pharmacol 7, 158-163 (2007). DOI: 10.1016/j.coph.2006.10.006. [PubMed: 17284359] 
(48). Carmeliet P VEGF gene therapy: Stimulating angiogenesis or angioma-genesis? Nat. Med 6, 1102-1103 (2000). DOI: 10.1038/80430. [PubMed: 11017137]

(49). Jiang X, Lin H, Jiang D, Xu G, Fang X, He L, Xu M, Tang B, Wang Z, Cui D, Chen F and Geng H Co-delivery of VEGF and bFGF via a PLGA nanoparticle-modified BAM for effective contracture inhibition of regenerated bladder tissue in rabbits. Sci. Rep 6, 1-12 (2016). DOI: 10.1038/srep20784. [PubMed: 28442746]

(50). Sun XT, Ding YT, Yan XG, Wu LY, Li Q, Cheng N, Qui YD and Zhang MY Angiogenic synergistic effect of basic fibroblast growth factor and vascular endothelial growth factor in an in vitro quantitative microcarrier-based three-dimensional fibrin angiogenesis system. World J. Gastroenterol 10, 2524-2528 (2004). DOI: 10.3748/wjg.v10.i17.2524. [PubMed: 15300897]

(51). Briganti E, Lisella A, Sanguinetti E, Soldani G, Losi P, Errico C and Chiellini F Fibrin-based scaffold incorporating VEGF- and bFGF-loaded nanoparticles stimulates wound healing in diabetic mice. Acta Biomater. 9, 7814-7821 (2013). DOI: 10.1016/j.actbio.2013.04.019. [PubMed: 23603001]

(52). Yoshiji H, Kuriyama S, Yoshii J, Ikenaka Y, Noguchi R, Hicklin DJ, Huber J, Nakatani T, Tsujinoue H, Yanase K, Imazu H and Fukui H Synergistic effect of basic fibroblast growth factor and vascular endothelial growth factor in murine hepatocellular carcinoma. Hepatology 35, 834842 (2002). DOI: 10.1053/jhep.2002.32541. [PubMed: 11915029]

(53). Pepper MS, Ferrara N, Orci L, Montesano R. Potent synergism between vascular endothelial growth factor and basic fibroblast growth factor in the induction of angiogenesis in vitro. Biochem Biophys Res Commun. 189:824-831(1992). [PubMed: 1281999]

(54). Mignatti P, Ren CJ, Rifkin DB, Robbins ES, Pintucci G, Seghezzi G, Gualandris A, Shapiro RL, Patel S and Galloway AC Fibroblast Growth Factor-2 (FGF-2) Induces Vascular Endothelial Growth Factor (VEGF) Expression in the Endothelial Cells of Forming Capillaries: An Autocrine Mechanism Contributing to Angiogenesis. J. Cell Biol 141, 1659-1673 (2002). DOI: 10.1083/ jcb.141.7.1659.

(55). Mandriota SJ \& Pepper MS Vascular endothelial growth factor-induced in vitro angiogenesis and plasminogen activator expression are dependent on endogenous basic fibroblast growth factor. J. Cell Sci 110 Pt 1, 2293-2302 (1997). [PubMed: 9378778] 
A

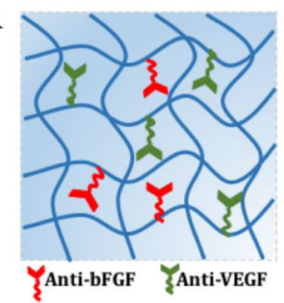

B

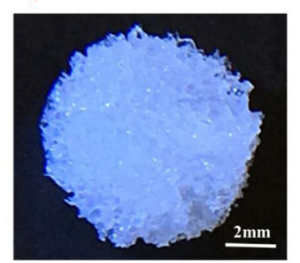

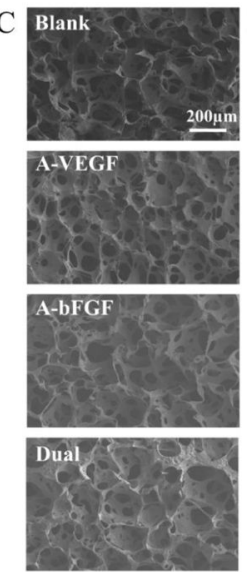
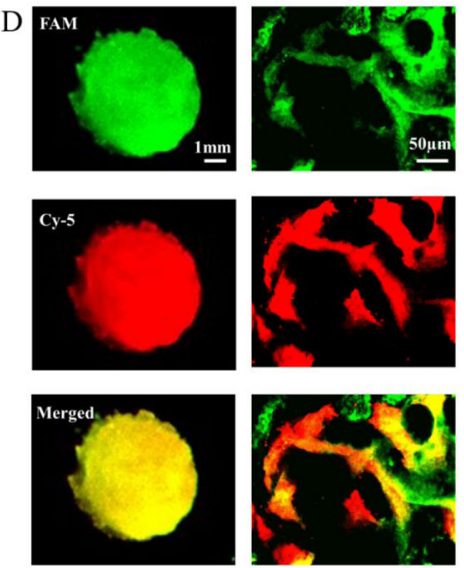

Fig. 1.

Synthesis and characterization of dual aptamer-functionalized macroporous hydrogels. A. Schematic illustration. B. Photographic image of the entire hydrogel. C. SEM images. Blank: hydrogel without aptamers; A-VEGF: anti-VEGF aptamer-functionalized hydrogel; A-bFGF: anti-bFGF aptamer-functionalized hydrogel; Dual: hydrogel functionalized with both anti-VEGF and anti-bFGF aptamers. D. Fluorescence (left) and confocal (right) microscopy images. The anti-VEGF and anti-bFGF aptamers were stained with FAM and Cy-5 labeled complementary sequences of the two aptamers, respectively. 


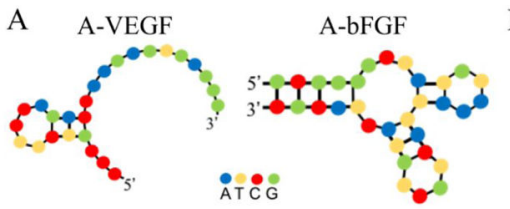

B

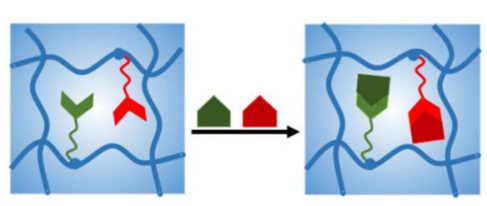

$\mathrm{C}$

$\mathrm{D}$
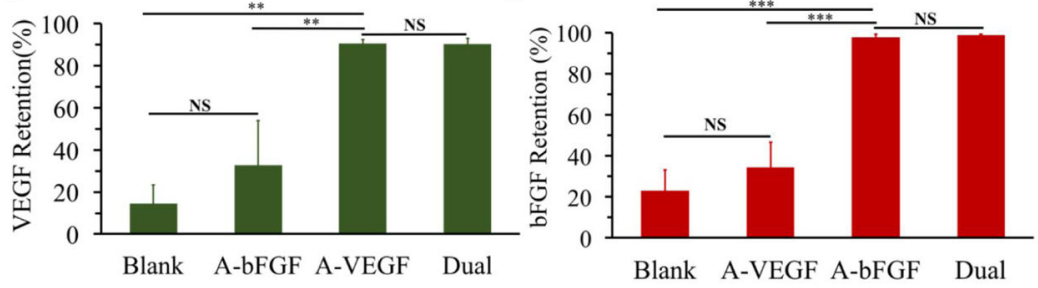

Fig.2.

Growth factor sequestration in aptamer-functionalized macroporous hydrogel. A) Secondary structure of anti-VEGF (A-VEGF) and anti-bFGF (A-bFGF) aptamers. B) Schematic illustration of growth factor loading and binding in aptamer-functionalized macroporous hydrogel. C) VEGF retention in macroporous hydrogels. D) bFGF retention in macroporous hydrogels. Blank: macroporous hydrogel without aptamers. Dual: macroporous hydrogel functionalized with both A-VEGF and A-bFGF aptamers. (NS: Non-significant. ** $\mathrm{p}<0.01$, $* * * \mathrm{p}<0.001)$. 
A
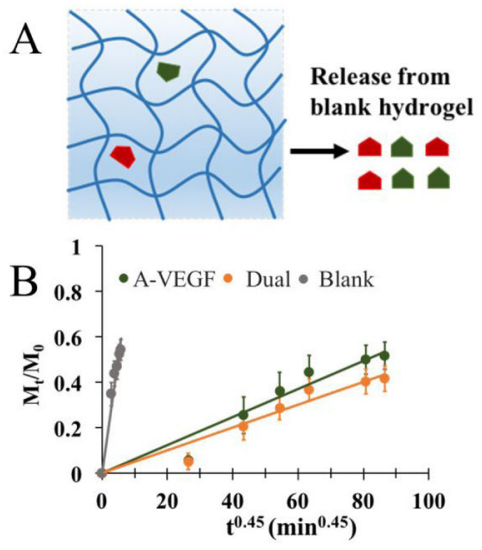

$\mathrm{C}$

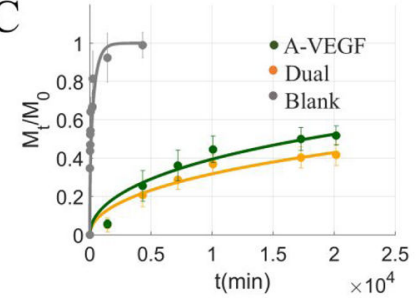

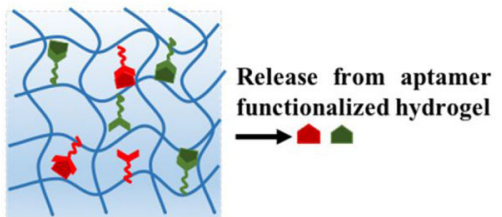
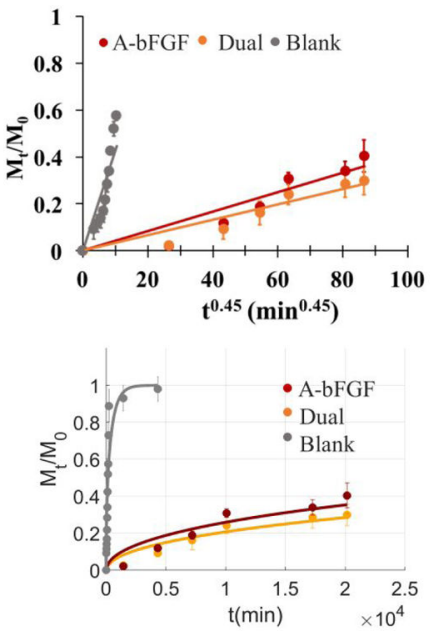

Fig. 3.

Examination of growth factors release from aptamer-functionalized macroporous hydrogels. A) Schematics showing the release of VEGF and bFGF from blank hydrogel versus from aptamer-functionalized hydrogels. B) Cumulative VEGF and bFGF release and data fitting based on Equation 2. C) Cumulative VEGF and bFGF release and data fitting based on Equation 3. 
A Diffusivity of growth factors $\left(\mathrm{cm}^{2} / \mathrm{sec}\right)$

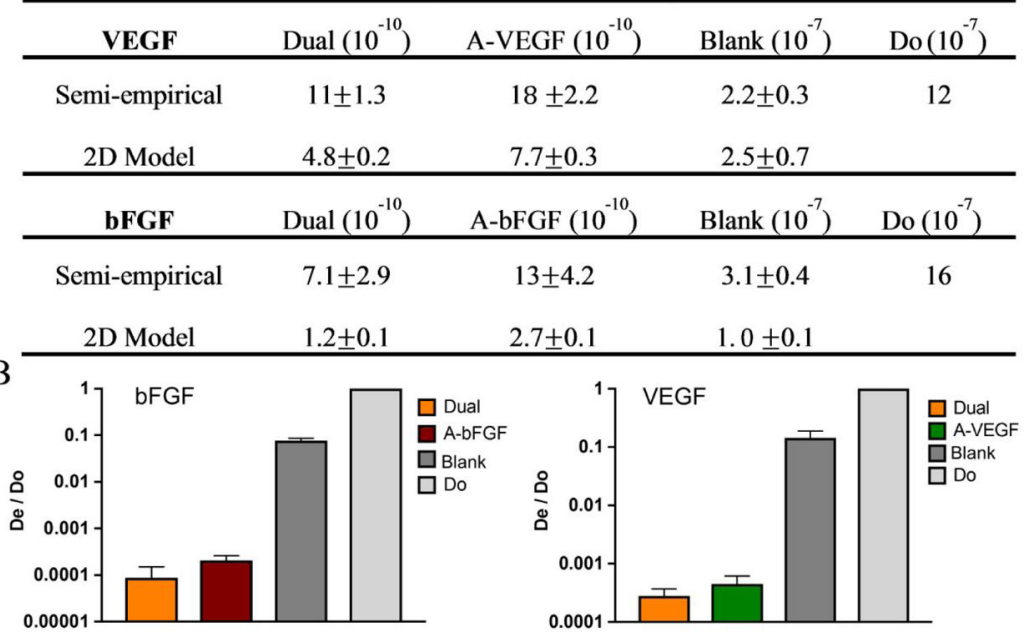

Fig. 4.

Comparison of apparent diffusivity. A) A summary of calculated diffusivity for bFGF and VEGF released from blank, single aptamer-functionalized and dual aptamer-functionalized macroporous hydrogels. B) Normalized apparent diffusivity. The calculated apparent diffusivity was divided by diffusivity of growth factors in aqueous solution (Do) for normalization. 


\author{
A
}

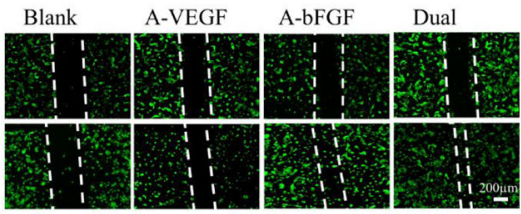

C

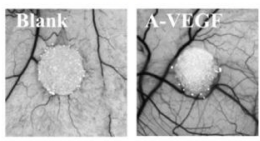

E

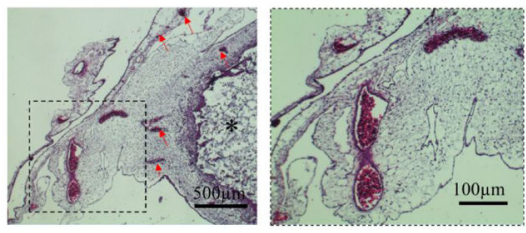

B

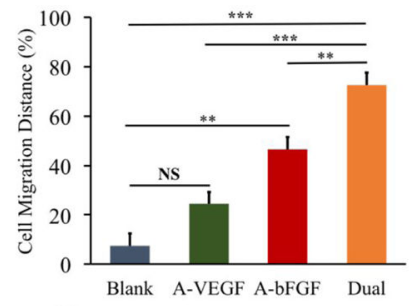

D

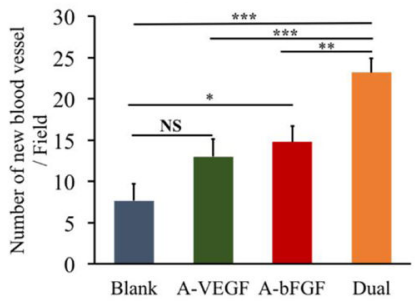

Fig. 5.

Evaluation of dual aptamer-functionalized macroporous hydrogel in stimulating angiogenic response in vitro and in vivo. Hydrogels were incubated in the release medium for 3 days to mimic a potential in vivo release microenvironment before conducting the studies. A)

Visualization of cell migration before and after treatment for all groups. The cells were stained with Calcein AM. B) Quantitative analysis of cell migration distance. C) Image of CAM at day 3 after implantation of hydrogels (Embryonic day 11). D) Quantitative analysis of new blood vessels formed after treatment. E) H\&E staining of CAM treated with dual aptamer-functionalized hydrogels loaded with VEGF and bFGF (Red arrows point at small blood vessels. * hydrogel). Blank: hydrogel without aptamers (VEGF and bFGF loaded); AVEGF: anti-VEGF aptamer-functionalized hydrogel (VEGF loaded); A-bFGF: anti-bFGF aptamer-functionalized hydrogel (bFGF loaded); Dual: hydrogel functionalized with both anti-VEGF and anti-bFGF aptamers (VEGF and bFGF loaded). (NS- Non-significant, $* \mathrm{p}<0.05, * * \mathrm{p}<0.01, * * * \mathrm{p}<0.001)$. 